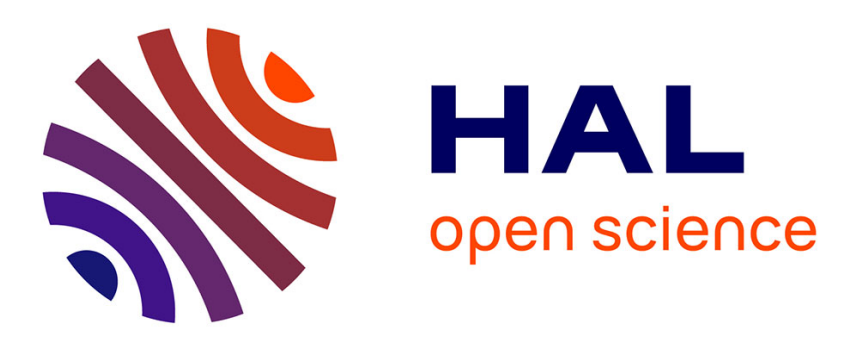

\title{
Circulation des objets et élaboration des relations dans les cérémonies du nord de la Kanaky Nouvelle-Calédonie
}

Denis Monnerie

\section{To cite this version:}

Denis Monnerie. Circulation des objets et élaboration des relations dans les cérémonies du nord de la Kanaky Nouvelle-Calédonie. Archimède: archéologie et histoire ancienne, 2020, 7, pp.96-113. halshs-02893178

\section{HAL Id: halshs-02893178 \\ https://shs.hal.science/halshs-02893178}

Submitted on 8 Jul 2020

HAL is a multi-disciplinary open access archive for the deposit and dissemination of scientific research documents, whether they are published or not. The documents may come from teaching and research institutions in France or abroad, or from public or private research centers.
L'archive ouverte pluridisciplinaire HAL, est destinée au dépôt et à la diffusion de documents scientifiques de niveau recherche, publiés ou non, émanant des établissements d'enseignement et de recherche français ou étrangers, des laboratoires publics ou privés. 


\section{ARCHIMĖDE N N 7}

DOSSIER THÉMATIQUE :

GESTES RITUELS. DE LA TRACE À L'INTERPRÉTATION

1 Sylvie DONNAT, Jean-Marie HUSSER

Le rite et ses traces. Perspectives méthodologiques

6 Marie AUGIER

Corps et objets interdits dans les sanctuaires (monde grec, ve av. - III ${ }^{\mathrm{e}}$ ap. J.-C.)

23 Claire CAMBERLEIN

Offrir l'antique à la divinité. L'exemple de l'adyton du sanctuaire de Vryokastro sur l'île de Kythnos (Cyclades)

37 Sylvie DONNAT

Les gestes rituels autour des papyrus-amulettes (Égypte, fin du II millénaire av. n. è.)

51 Jean-Marie HUSSER

Entre rituel, mémorial et littérature de propagande. À propos de deux textes ougaritiques au genre mal défini

62 Anne JACQUEMIN

Rituels efficaces pour fonder ou déplacer un culte en Grèce ancienne

76 Françoise LAROCHE-TRAUNECKER

Les rituels de construction des temples égyptiens. Gestes représentés sur les parois et actes attestés par des fouilles à Karnak

96 Denis MONNERIE

Circulation des objets et élaboration des relations dans les cérémonies du nord de la Kanaky Nouvelle-Calédonie

\section{ACTUALITÉ DE LA RECHERCHE}

294 VARIA 


\title{
CIRCULATION DES OBJETS ET ÉLABORATION DES RELATIONS DANS LES CÉRÉMONIES DU NORD DE LA KANAKY NOUVELLE-CALÉDONIE
}

\author{
Denis MONNERIE \\ Professeur d'ethnologie \\ Université de Strasbourg \\ UMR 7367 DynamE \\ monnerie@unistra.fr
}

\section{RÉSUMÉ}

Au nord de la Grande Terre de Kanaky NouvelleCalédonie (Océanie), d'amples cérémonies, élaborent périodiquement les relations sociales et au monde. Elles sont des institutions du monde kanak pouvant mobiliser des centaines de personnes. Ce sont principalement des cérémonies du cycle de vie des personnes et des cérémonies entre des entités sociales localisées. Un modèle anthropologique qui fait ressortir l'ensemble des dynamiques de ces cérémonies et met en avant le concept d'ensemble cérémoniel permet de dégager quelques-uns de leurs principes. L'un d'eux est, dans un cadre dyadique, la mise en œuvre de transferts et circulations d'objets spécifiques, toujours synchronisés avec des discours cérémoniels, qui tressent et nouent les relations. Ceci permet de suggérer les contrastes entre cette approche et certaines théories des échanges et des rituels. Dans ce texte, ce modèle est mis à contribution pour réfléchir à la place des objets cérémoniels à travers leurs circulations, leurs significations, les articulations aux discours et aux relations qu'ensemble ils élaborent pendant le déroulement de ces cérémonies. circulation,

modèle anthropologique.
In the North of Kanaky New Caledonia's Grande Terre (Oceania), large ceremonies which periodically elaborate relations which are at once social and cosmic, and which then will prevail in everyday life. These institutions of the Kanak world can be attended by hundreds of people. They are mainly cycle of life ceremonies and ceremonies dealing with local social entities. I outline an anthropological model which highlights the dynamics of these ceremonies and puts forward the concept of ceremonial set, which is aimed at understanding some of the ceremonial principles. One of these is, within a dyadic framework, the use of specific forms of transfers and circulations of specific objects, always synchronised with ceremonial speeches, in order to plait and tie relations. This suggests several contrasts between this approach and some theories of rituals or exchanges. In this text, this model is mainly put forward to reflect upon the role of ceremonial objects, through their circulations, their meanings, their articulations to verbal discourse and towards the relations which, together, they elaborate during the course of these ceremonies.
KeYWORDS

Oceania, exchange, ritual, circulation, anthropological model. 
Ce texte traite des cérémonies contemporaines des Kanak [1] de l'extrême nord de Nouvelle-Calédonie [2]. Colonie française [3] depuis 1853, cet archipel, centré sur une Grande Terre de plus de $400 \mathrm{~km}$ de longueur, se situe à l'ouest de l'Océanie, région connue comme Mélanésie. On y parle 28 langues austronésiennes ainsi que diverses formes du français.

La présentation qui suit concerne une série de questions autour des cérémonies kanak et des objets qui y circulent - les objets cérémoniels. Elle s'appuie sur un modèle anthropologique de ces cérémonies que je conçois comme une proposition pour la description et les analyses comparatives de celles pratiquées dans d'autres parties de Kanaky et ailleurs en Océanie [4] et Indonésie.

Souvent invoquées par les Kanak comme les définissant, les cérémonies sont une de leurs préoccupations majeures. Elles peuvent mobiliser des centaines de participants lors de mariages, de funérailles et de grandes cérémonies régionales. Elles sont généralement décrites comme cérémonies, ou rituels d'échanges. En raison du thème de cette livraison $\mathrm{d}^{\prime}$ Archimède, dans cet article, ma réflexion sera pondérée sur le deuxième terme de ces définitions, pour lequel je vais montrer que le concept de circulation est plus pertinent que celui d'échanges que nous héritons de I'histoire de l'ethnologie [5].

L'importance des échanges en Océanie a été très vite soulignée par les pionniers de l'ethnographie en Mélanésie, R. Thurnwald [6] et B. Malinowski [7], suivis par M. Mauss [8] qui, ayant étendu sa réflexion à la Polynésie et à d'autres parties du monde a proposé

[1] Depuis les années 1980 les descendants des océaniens précoloniaux se désignent par ce terme, invariable, nommant Kanaky l'ensemble de leur pays. Merci à mes innombrables hôtes kanak pour leur hospitalité pendant mes terrains ethnographiques de 1992 à 2017. C'est à cette période que je réfère dans mon utilisation du présent ethnographique.

[2] Une version récente de ces recherches a été présentée au Séminaire du Transversal II du laboratoire UMR 7044 Archimède « Gestes rituels : traces matérielles et interprétations. Archéologie, histoire, sciences sociales », à l'Université de Strasbourg, le 23 mai 2016. Pour leurs suggestions, discussions, lectures ou références concernant diverses versions de ces recherches, je voudrais remercier I. Berdah, P. Bonnemère, S. Caillon, S. Chave-Dartoen, U. Cugola, A. Clerc-Renaud, D. Fasquel, P. Lemonnier, J.D.M. Platenkamp et J.M. Servet. Merci à Chloé le Mouël qui a réalisé les figures. Les prises de positions et éventuelles erreurs sont bien sûr de mon seul fait. une théorie du «don ». Ses interrogations sont aujourd'hui encore au cœur d'importants débats des sciences sociales qui portent sur la place des échanges, de la réciprocité, du don, de la dette dans les sociétés, en dialogue implicite ou explicite avec les théories de l'économie euro-américaine. Alors que je m'appuie sur ces recherches et débats, mes analyses de l'ethnographie m'amènent à interroger les notions même d'échanges, de don et de dette [9].

Dans une perspective privilégiant les conceptions émiques (emic), les exigences de rigueur de l'ethnographie analytique impliquent de décrire, théoriser et modéliser les pratiques observées au plus près de leurs réalités et conceptualisations locales. Si on préfère, un de mes objectifs est - autant que faire se peut [10] - d'éviter de décrire les cérémonies, éléments clés de socialité du monde kanak, avec le vocabulaire et l'idéologie importés en Kanaky depuis le XIXe siècle par la colonisation [11].

\section{LES CÉRÉMONIES KANAK}

En Kanaky Nouvelle-Calédonie, mes recherches portent sur la partie septentrionale de la Grande Terre. Un ensemble régional qui préexistait à la colonisation, nommé Hoot ma Whaap y regroupe une douzaine de sociétés dont la moitié, désignées comme Hoot, sont considérées comme d'implantation plus ancienne que les sociétés Whaap. Ce réseau régional acéphale avait autrefois des expressions guerrières où s'affrontaient des alliances Hoot et Whaap. Il avait aussi des

[3] Aux statuts changeants, voir LeBLIC 1993, GRAFF 2015.

[4] Avec S. Chave-Dartoen, dans une démarche comparatiste, nous étudions les continuités et discontinuités avec celles pratiquées à Wallis (Polynésie occidentale); voir Chave-Dartoen \& MonNerie, à paraître.

[5] D'autres aspects des cérémonies kanak seront étudiés dans d'autres textes, ou l'ont été : MonNeriE 2005, 2014a.

[6] THURNWALD 1937.

[7] MALINOWSKI 1922.

[8] MAUSS 1923-1924.

[9] MONNERIE 2014b.

[10] DUMONT 1978, p. 83-100.

[11] MONNERIE 2018. 
expressions pacifiques, en particulier cérémonielles, qui perdurent [12]. Mes terrains ethnographiques ont été centrés sur la société d'Arama [13], à l'extrême nord-est qui s'étend sur une dizaine de kilomètres entre des montagnes arides et une côte frangée de mangrove. Y vivent un demi-millier d'habitants kanak, horticulteurs et pêcheurs avec, pour certains, de façon irrégulière, un accès à des emplois salariés [14].

Le vocabulaire colonial et le français kanak désignent les cérémonies kanak comme « coutumes », terme fourre-tout qui s'applique à peu près à tout ce qui concerne le monde kanak et dont il faut se débarrasser pour en approcher une compréhension anthropologique. Localement, les connaisseurs, eux, parlent de «système kanak ». C'est sa variante pratiquée à Arama et au nord de Hoot ma Whaap, qui est modélisée ici. De surcroît, je ne saurais assez souligner que ces cérémonies, qui seules me retiennent dans ce texte, ne sont pas le tout de la vie sociale kanak. Il existe d'autres régimes de socialité, dont la vie courante, les marchés hebdomadaires, les relations avec l'église catholique, les relations politico-administratives, les relations avec l'économie, etc. [15].

La forme cérémonielle simple opère des arrivées et des accueils de quelques personnes. Les cérémonies complexes sont déclenchées par des décisions collectives ou des événements - année horticole, décès. Elles peuvent mobiliser les participants en plus ou moins grand nombre. Une grande distinction heuristique peut être posée entre deux sortes de cérémonies complexes. D'une part les cérémonies du cycle de vie des personnes, il s'agit aujourd'hui principalement des mariages et des funérailles. D'autre part les cérémonies des entités sociales localisées [16] : cérémonies annuelles de prémices des ignames et cérémonies régionales.

Le modèle que je propose vise à rendre compte de ces différents types de cérémonies, dont les enjeux sont la réaffirmation et/ou la transformation des relations sociales et au monde. En effet, dans leur ensemble, ces cérémonies peuvent être abordées sous un angle simple mais scientifiquement productif : ce sont des procédures collectives d'élaboration des relations sociales et au monde [17]. Par « élaborer », j'entends (ré)affirmer, (re)vivifier, (ré)activer, transformer, exprimer, créer. Participent à ces cérémonies une grande partie des personnes, ensembles sociaux, non humains et espaces concernés par l'élaboration de relations qui sont ainsi sociales et cosmiques. Celles-ci valent ensuite dans la vie courante, jusqu'à ce que soient tenues d'autres cérémonies impliquant ces relations à quelque degré. Dans un monde kanak où les relations sont considérées comme vulnérables et doivent périodiquement être réactivées, les cérémonies sont des institutions sociales centrales. Elles se caractérisent par leur forme dynamique et leur transmission dite « orale »- qui associe en fait des dimensions intellectuelles, pluriperceptuelles et plurisensorielles [18] - ce qui en fait des institutions avec des traits caractéristiques des mondes océanien et indonésien, mais qui sont fort éloignées de celles des mondes européens qui nous sont familiers.

\section{LES CÉRÉMONIES KANAK, ÉTAT DE L'ART}

Pour la Grande Terre, les premiers textes sur la Nouvelle-Calédonie évoquent [19] ou décrivent [20] des cérémonies, et on en possède quelques images. Toutes ces sources montrent leur importance dans les pratiques sociales kanak. Mais, incomplètes et peu détaillées, les données ne se prêtent pas à des analyses anthropologiques modernes. Il faut attendre la fin du $\mathrm{XX}^{\mathrm{e}}$ siècle pour que soient publiées dans ce domaine des propositions solides répondant aux critères scientifiques qui, en Océanie, prévalent dans les recherches, beaucoup plus avancées sur ces questions, en particulier pour la Nouvelle-Guinée et les Iles Salomon. Pour les cérémonies kanak, l'analyse pionnière de Patrick Pillon [21] porte sur les cérémonies funéraires. Isabelle Leblic [22], Charles Illouz [23] et Patrice Godin [24] ont analysé des aspects importants
[12] Depuis les années 1990 elles sont doublées de relations administratives dans le cadre du Conseil coutumier Hoot ma Whaap, rattaché au Sénat coutumier Kanak.

[13] Les termes vernaculaires de ce texte sont dans la langue de cette société, le nyêlâyu d'Arama.

[14] Pour une présentation plus complète, MoNNERIE 2005 ; pour le réseau Hoot ma Whaap, MonNerIE $2016 \mathrm{~b}$; pour les distorsions apportées par les pratiques et catégories coloniales, MoNNERIE 2018.

[15] MONNERIE 2008.
[16] Surtout hameaux et sociétés locales.

[17] Ci-après : élaboration des relations.

[18] MONNERIE 2014a.

[19] COOK 1780.

[20] LAMBert 1900, Gagnère 1905, LeEnhardT 1937, p. $159-170$.

[21] PILLON 1995.

[22] LEBLIC 2004, 2008, p. 209-214, 2010.

[23] ILLOUZ 2010.

[24] GODIN 2005. 
de diverses cérémonies. Actuellement aucun ouvrage ne décrit les mariages de façon approfondie [25]. Deux ouvrages décrivent et analysent de façon détaillée de grandes cérémonies kanak et en montrent la complexité. Dominik Bretteville [26] étudie des cérémonies funéraires, à Paimboa au centre nord de Hoot ma Whaap. Mon propre livre [27] porte sur les grandes cérémonies translocales de la même région. Je me distingue de tous ces auteurs qui privilégient les cérémonies focales car j'englobe leur étude dans celle des ensembles cérémoniels qui les préparent et les concluent (voir infra).

\section{UN MODĖLE POUR LES CÉRÉMONIES KANAK}

L'observation ethnographique montre que les cérémonies se caractérisent par des amplitudes très différentes en termes de nombre de participants, de développements spatio-temporels, des objets mis en circulation, des repas collectifs, mais aussi par le fait que toutes partagent certains principes communs. Mon modèle considère les formes cérémonielles simples comme permettant de mettre au jour ces principes. L'un d'entre eux est la forme dyadique de toutes les cérémonies, avec deux côtés cérémoniels qui se font face et où des hommes élaborent des relations en transférant entre eux des prestations d'objets et de paroles (fig. 1). Un autre principe est la conclusion de ce face à face relationnel par le partage d'un repas préparé par des femmes. Je reviendrai sur ces principes et leur développement dans les cérémonies complexes.

Ce modèle, avec les schémas qui le visualisent, est une épure qui privilégie les éléments cérémoniels récurrents les plus significatifs concernant les circulations de personnes et d'objets. Il implique une déperdition dans les données ethnographiques, un aspect de la modélisation qui est classique en anthropologie

Figure 1

Modèle graphique de la forme cérémonielle minimale.

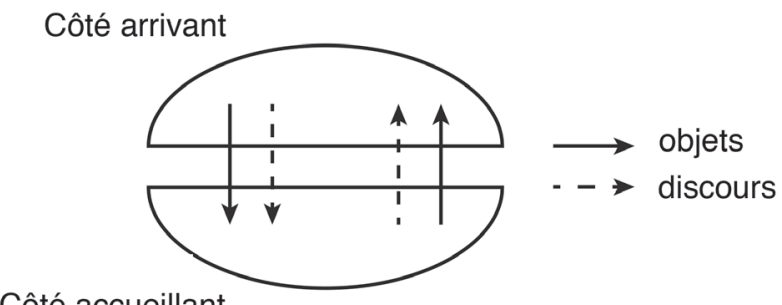

Côté accueillant
- en particulier en études de parenté [28]. Ceci bien entendu, est aussi de façon générale un trait central de la notion même de modèle scientifique.

Comme dans les travaux de R. Hertz (1908), A. Van Gennep (1909) et V. Turner (1968), mes précédentes analyses de cérémonies impliquaient une perspective séquentielle, mettant au premier plan I'analyse du déroulement des cérémonies et leur découpage en segments [29]. Je propose désormais une approche plus serrée en termes de procédures opératoires, distinguant les opérations, séquences, phases, étapes et périodes d'un ensemble cérémoniel [30]. Ceci permet d'articuler avec plus de précision les participations, positions, actes, dynamiques de circulation des objets et personnes avec leurs places relatives et significations dans l'élaboration des relations.

Ces principes généraux étant posés, je présenterai les formes cérémonielles simples, puis les formes complexes.

\section{FORME CÉRÉMONIELLE MINIMALE ET FORMES SIMPLES}

La forme minimale des cérémonies kanak opère l'arrivée et l'accueil d'un homme dans une localité qui n'est pas la sienne. L'arrivant a fait prévenir l'accueillant, un dignitaire local, pour convenir du lieu et du moment de leur rencontre.

L'arrivant dépose au sol une prestation de nourriture, idéalement ignames et taros de son pays. Il tient à la main et présente un billet plié enveloppé dans une étoffe. Il va donner cette prestation manuelle et celle de nourriture à I'homme qui I'accueille, synchronisant ses gestes de transfert avec la fin d'un discours qu'il prononce pour expliquer le contexte et

[25] Une description, sans analyse, par GUIART 1992, p. 304-321 de segments d'un mariage à Lifou (Iles Loyauté) suffit à donner une idée de la complexité de ces cérémonies qui diffèrent sensiblement de celles du nord de Hoot ma Whaap.

[26] BRETTEVILLE 2019.

[27] MONNERIE 2005.

[28] Il est intéressant du point de vue méthodologique que I'analyse des cérémonies funéraires par Patrick Pillon ait été appuyée sur des schémas de parenté, ce qui résulte en un modèle très épuré (PILLON1995). Voir aussi LEBLIC 2004.

[29] MONNERIE 1996 pour Mono-Alu aux Iles Salomon ; MONNERIE 2005 pour les grandes cérémonies thiam du nord de Hoot ma Whaap.

[30] Mes références théoriques sont LEROI-GoURHAN 1965, COPPET 1981, LemONNIER 2005, COUPAYE 2013. 
la portée de leurs rencontre et relations. L'accueillant I'écoute et prend ces prestations. Après cette première séquence de transfert, en une séquence de contre-transfert [31], il répond par un discours d'accueil précisant son point de vue sur la rencontre et les relations, tout en tenant à la main puis en donnant à son vis-à-vis sa propre prestation de monnaie enveloppée d'étoffe (fig. 1). Pendant ce temps, dans une cuisine voisine, une femme proche de l'accueillant a préparé une collation ou un repas. L'accueillant invite alors l'arrivant à partager cette nourriture. Du fait des relations ainsi élaborées avec le dignitaire accueillant, I'arrivant aura une large autonomie dans la localité d'accueil et avec ses habitants. Une cérémonie de séparation est généralement tenue quand I'arrivant quitte la localité d'accueil.

Cette forme cérémonielle d'élaboration des relations que je qualifie de minimale - qui fait se rencontrer et interagir au moins deux hommes et une femme se développe couramment en formes proches que je nomme formes simples. Celles-ci mobilisent quelques personnes du côté des arrivants et des accueillants et à la cuisine, mais elles mettent en œuvre une procédure d'élaboration des relations semblable à celle de la forme minimale, articulant les mêmes opérations et séquences cérémonielles entre les deux côtés et pour le repas final. Le plus souvent, dans les pages qui suivent, je traiterai de ces formes simples.

De nombreux éléments clés des cérémonies kanak ressortent de l'analyse de cette forme simple qui, à partir de rapports d'appartenance ou d'extériorité et d'altérité, formalisés dans la distinction dyadique entre accueillant et arrivant, réalise de façon dynamique l'intégration des deux côtés en un seul ensemble pour des durées pouvant varier. Élaborant la relation d'accueil et d'intégration par une suite d'opérations, trois séquences s'enchaînent de façon fluide.

(I) Une séquence de transfert de l'arrivant vers l'accueillant, composée d'opérations de présentation des prestations - distinguant prestations de nourritures posées au sol et prestation manuelle - d'énonciation de discours suivi des gestes de transfert de ces prestations qui sont prises par l'accueillant.

(II) Suivie immédiatement d'une séquence de contre-transfert de l'accueillant vers I'arrivant, synchronisant les opérations de présentation de sa propre prestation manuelle avec son discours, suivi du contre-transfert de cette prestation, prise par l'arrivant.

(III) La préparation puis le partage et l'ingestion d'une collation ou d'un repas.

Je nomme les séquences enchaînées (I) et (II) séquences de circulation des prestations et la séquence (III) séquence de validation des relations.
La notion, classique en anthropologie, de prestation cérémonielle est ici importante. En effet dans les prestations, les objets cérémoniels circulent toujours associés à d'autres. Les prestations et les trajectoires qui les portent sont nettement distinguées. Ici il s'agit de prestations composites de tubercules, de monnaie et étoffes (que je nomme prestations manuelles). Dans les cérémonies complexes ces distinctions sont encore plus raffinées. Je nomme prestations non verbales ces composites d'objets avec les gestes qui les présentent et les déplacent - tous sont porteurs de significations. Qui plus est, des discours, que je définis comme prestations verbales, sont étroitement synchronisés avec ces prestations non verbales et les commentent. Cette configuration de combinaison étroite de prestations non verbales et verbales est une des caractéristiques des cérémonies kanak [32].

Je vais maintenant détailler ce que le positionnement cérémoniel nous indique sur les partenaires établissant des relations, puis l'importance des gestes et discours de transfert et contre-transfert et enfin celle du partage du repas.

La dyade cérémonielle opère distinction et intégration par la dynamique d'arrivée et d'accueil qui rapproche un arrivant allochtone et un accueillant autochtone, elle implique en effet des hommes situés dans le monde. L'accueillant est dans son pays (phweemwâ), une notion qui, aux espaces, environnement et terroirs associe les vivants, les ancêtres, d'autres non humains et l'ensemble des noms qui les désignent. L'arrivant, lui, est venu par un chemin (daan), transportant et présentant des plantes de son propre pays. Ainsi, dans la scène cérémonielle dyadique, la mise en relations implique une performance aux multiples dimensions significatives implicites - déplacement, position, et gestes de chacun -, auxquelles se combinent les significations non verbales des objets transférés et celles, verbales des discours.

Dans les deux premières séquences, la monstration et la préhension des prestations attestent du sens et de la vérité de ce qui est transféré. Ceci est exprimé dans un mythe (vajama) qui souligne l'importance de « tenir (cuva) avec la main (yen) pour que les yeux (thevân) voient (kiia) ». Sur un plan non verbal, ces actes constituent (thu) le sens, la vérité (paer) des cérémonies et des relations qu'ils expriment. Ces attestations portent

[31] J'emploie les termes transfert et contre transfert pour décrire des translations d'objets - pas au sens psychanalytique.

[32] Voir MONNERIE 2005 
à la fois sur les objets composant les prestations et sur les gestes de leurs manipulations. Dans les séquences en aller-retour des transfert et contre-transfert, les opérations sont des actes dessinant graphiquement la trajectoire des relations en cours d'élaboration entre les deux partenaires. Ils visualisent et rendent préhensibles, palpables - donc vraies - les relations élaborées par ces gestuelles prescrites, comprises de tous, pérennes, en un mot, instituées.

L'implication des objets cérémoniels dans cette élaboration des relations lie de façon indissociable deux séries de faits. D'une part ils sont des substances matérielles dont les manipulations par les participants sont des gestes de nouage, de tressage. D'autre part ils sont les supports et les attestations des relations élaborées par ces actes. Outre cette absence d'opposition tranchée entre relations et substances [33], qui doit être associée à leur agentivité (agency [34]), les objets cérémoniels circulants ont aussi des dimensions sémiotiques [35] sur lesquelles je reviendrai.

L'ensemble de ces actes sont dits « accomplir » (thilin) la cérémonie, un terme qui signifie « assembler » et renvoie aux concepts de lien et de nouage. Mot-clé de cette société, wove, lui, désigne le geste de prendre une prestation cérémonielle, et signifie aussi « accueillir, tresser » ; il décrit des phases des mariages et des adoptions, rassemblant ainsi les cérémonies, la technique et le social en un large concept commun. Celui-ci implique une composition de plusieurs sortes d'objets avec des gestes prescrits, opérations qui constituent diverses sortes de brins relationnels visibles, palpable et intelligibles et les mettent en mouvement. L'élaboration des relations est ainsi un « nouage » et un « tressage » cérémoniel aux brins constitutifs démultipliés, fabriquant et exprimant à la fois de la robustesse, de la redondance, de la complexité, de la complétude et diverses formes d'expression de significations. Les relations élaborées par les cérémonies sont des composites constitués non verbalement et verbalement.

Car simultanément, les discours synchronisés avec ces gestes explicitent ces relations au plan verbal. Six principes généraux importent ici : 1) ces discours ponctuent les opérations cérémonielles de transferts et contre-transferts ; 2) ils ont une forme scandée ; 3) ils explicitent et développent verbalement certaines des significations non verbales des gestes et objets cérémoniels ; 4) ils verbalisent les relations entre les deux côtés de la dyade : le discours de transfert ouvre sur les relations, celui de contre-transfert y répond et les reconnaît ; 5) ils enveloppent I'élaboration des relations par leurs descriptions, significations et exaltations poétiques - dont le flou même peut laisser le champ ouvert à des débats ; 6) ils sont traversés par des tensions entre ce qui est explicite et verbalisé et les dimensions implicites de la cérémonie, de son cadre (voir supra) et de son contexte.

Pendant le déroulement de la scène cérémonielle masculine, la préparation et la cuisson des nourritures construisent des apports cérémoniels plus spécifiquement féminins qui préparent et permettent I'ultime séquence, celle de validation des relations. Celle-ci est souvent négligée dans les analyses de cérémonies, mais on ne saurait assez en souligner l'importance. Dans cette troisième et dernière séquence, le partage et l'ingestion par les commensaux des nourritures cuites par une ou plusieurs femmes du côté accueillant parachèvent ce processus d'élaboration des relations. Le repas, en effet, est un apport roboratif de « satiété » et de « vie »- le même mot, maôlep, désignant ces deux réalités [36].

L'étude en termes de procédure opératoire de la configuration minimale des cérémonies fait ainsi ressortir la place dans l'élaboration des relations d'articulations dynamiques entre appartenance et altérité. Ceci est effectué par des formes prescrites de médiations où les manipulations et transferts d'objets, des discours et le partage de nourriture jouent des rôles considérables et ont des significations précises. Ces traits des cérémonies kanak fondent l'analyse de leurs formes les plus complexes.

\section{UN MODĖLE POUR LES FORMES CÉRÉMONIELLES COMPLEXES}

Tous ceux qui les ont observées ou qui y ont participé ont remarqué la complexité, la solennité et l'importance des cérémonies kanak.

Les cérémonies complexes démultiplient la configuration simple que je viens de décrire pour composer un ensemble cérémoniel.

La meilleure façon de présenter de manière succincte les cérémonies complexes est d'en faire une première description, privilégiant ses développements formels dans l'espace et le temps et de la compléter par une seconde, du point de vue de ses dynamiques de

[33] MONNERIE 2016a.

[34] GELL 2009.

[35] PeirCe 1978.

[36] L'apport de tubercules crus d'ignames et de taros par l'arrivant, il faut le remarquer, ouvre à la fois sur de futurs repas, mais aussi éventuellement sur de futures plantations dans le pays d'accueil de boutures de ces tubercules très valorisés par la civilisation kanak, voir HAUDRICOURT 1964. 


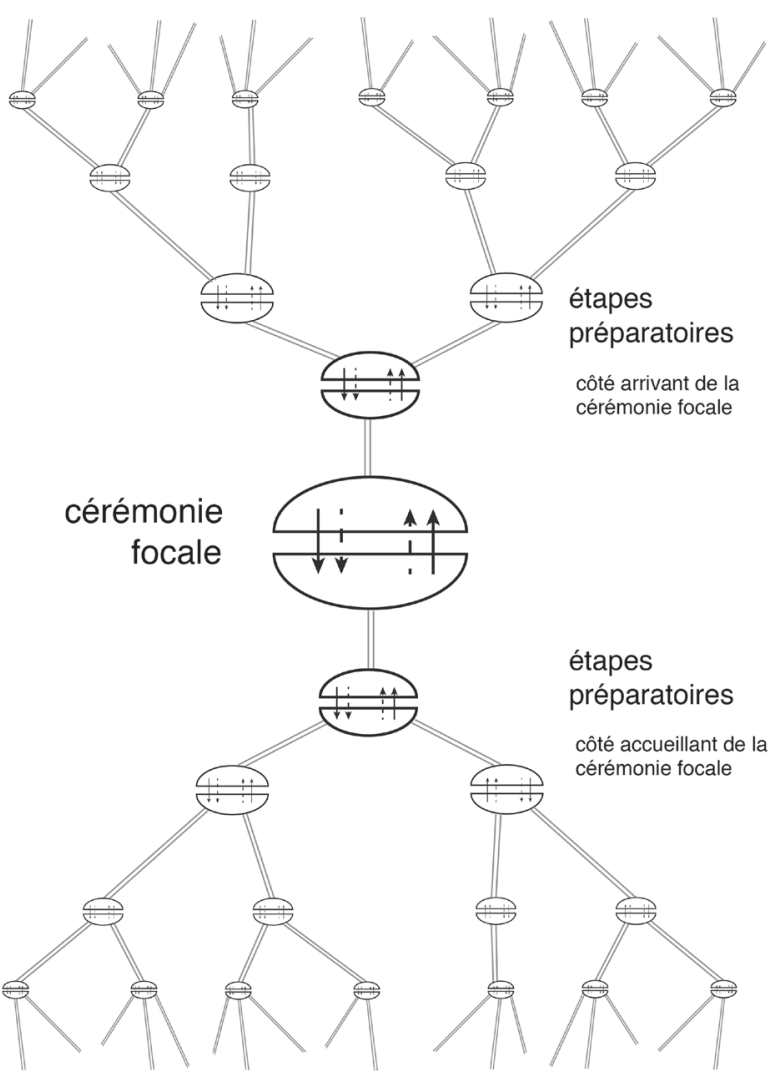

Figure 2

Modèle graphique des formes cérémonielles complexes.

circulations des participants et des prestations d'objets cérémoniels qu'ils y transportent. L'ancrage spatio-temporel d'un ensemble cérémoniel est la cérémonie focale, dont, après une décision concertée, la date et le lieu sont transmis par des messages. En fonction de cette donnée de base, chaque étape cérémonielle préparatoire [37] se tient elle-même dans un espace-temps décidé à l'avance.

Photo 1 : prestations pour un mariage à Gomen 2010, étape 1. Pour un petit collectif d'arrivants, un homme dispose au sol des prestations destinées à circuler vers la cérémonie focale. (c) Denis Monnerie.

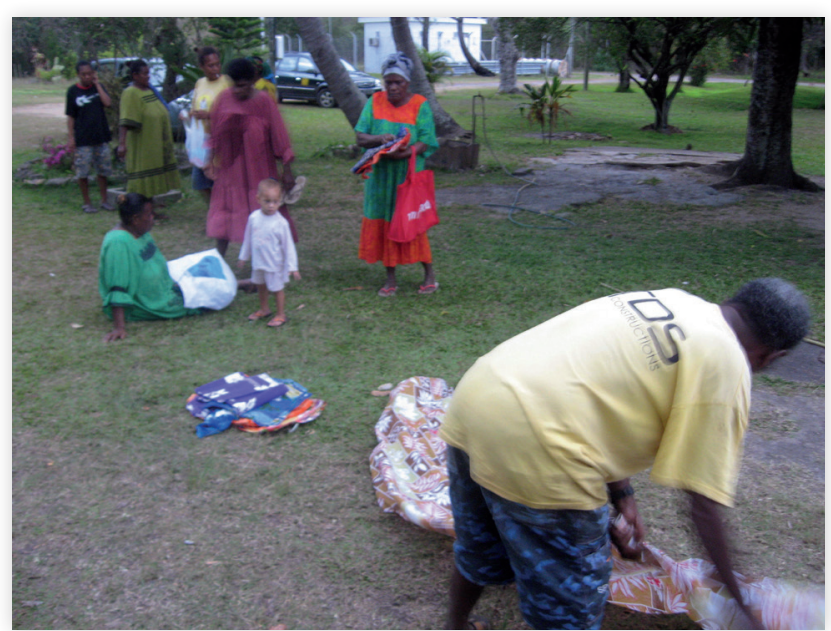

Dans les cérémonies complexes, le principe dyadique s'exprime de façon quasi fractale en ce qu'il organise (i) globalement l'ensemble cérémoniel, qui est constitué de deux sous-ensembles, (ii) eux-mêmes composés d'étapes cérémonielles de forme dyadiques, (iii) préparatoires à la cérémonie focale, elle aussi dyadique (fig. 2). Les étapes cérémonielles constituant les deux sous-ensembles qui préparent la cérémonie focale ont toutes une forme générale proche de la configuration cérémonielle simple. Mais leur ampleur est de plus en plus grande au fur et à mesure qu'elles se rapprochent de la cérémonie focale. À terme, ces deux sous-ensembles vont constituer les deux côtés qui se feront face dans la dyade de la cérémonie focale.

On peut prendre une autre vue des cérémonies complexes, celle des dynamiques de leurs circulations. De ce point de vue, la cérémonie focale est l'événement cérémoniel central, dyadique et à développement maximum, vers lequel convergent l'ensemble des circulations de personnes et d'objets cérémoniels, des deux sous-ensembles cérémoniels qui vont constituer les deux côtés de la cérémonie focale.

Dans chaque sous-ensemble, on passe d'une étape de niveau 1 (photo $\mathbf{1}$ ) vers des étapes d'ampleur croissante de niveaux 2, 3 et 4 (photo 2), etc. et composées de phases de plus en plus nombreuses, elles-mêmes comportant plusieurs séquences de circulations et

[37] Dans mon modèle, par convention, quatre étapes préparatoires à la cérémonie focale.

Photo 2 : prestations pour un mariage à Gomen 2010, étape 4. Au premier plan, les représentants des accueillants - le côté cérémoniel de l'époux - attendent qu'une des

vagues d'arrivants (au fond) aient fini de décharger et de disposer au sol leurs prestations destinées à circuler vers la cérémonie focale. Ensuite les deux côtés vont effectuer les séquences de transfert et contre-transferts. La cérémonie focale se tiendra le lendemain. (c) Denis Monnerie.

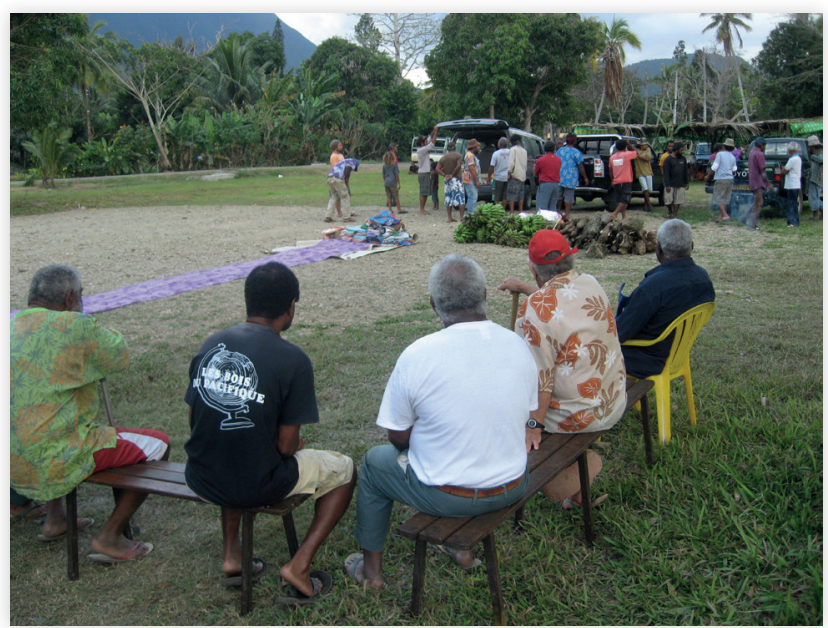


parachevées par un/des repas. S'y accumulent les prestations d'objets cérémoniels apportées par des groupes d'arrivants. Elles sont ensuite rassemblées, comptées. Puis les objets qui les composent sont triés et réorganisés pour constituer d'autres prestations. Celles-ci seront ensuite transportées par les accueillants vers la prochaine étape et d'autres prestations apportées et transférées par d'autres arrivants s'y ajouteront. Toutes seront rassemblées et seront, elles aussi, comptées, triées, réorganisées. Et ainsi de suite. Dans la période croissante, étape après étape, parallèlement à cette augmentation incrémentale du volume des prestations, la forme cérémonielle minimale se transforme elle aussi de façon incrémentale, avec toujours plus de démultiplications et d'intensifications des opérations, séquences et phases du processus opératoire, jusqu'à la cérémonie focale (fig. 2 ).

Ceci dans une première période, croissante qui est suivie à partir de la fin de la cérémonie focale de redistributions de prestations de retour qui constituent la seconde période, décroissante, de ces deux sous-ensembles.

Je modélise ainsi les cérémonies kanak en montrant I'aspect crucial d'une réflexion fondée sur un matériau ethnographique qui ne se limite pas à la seule cérémonie focale, mais inclut les cérémonies préparatoires qui la rendent possible et les redistributions qui la suivent. Étudier un ensemble cérémoniel permet à la fois d'élargir, d'affiner et de renforcer la pertinence des descriptions et interprétations des cérémonies kanak.

\section{DYNAMIQUES INCRÉMENTALES DES FLUX DE PRESTATIONS D'OBJETS CÉRÉMONIELS}

Comment les objets cérémoniels sont-ils impliqués dans ces procédures [38] ? Dans leurs prestations, les arrivants en transfèrent toujours plus que les accueillants n'en contre-transfèrent pour attester des relations élaborées. En effet, dans les séquences de circulation, les arrivants transfèrent les prestations qu'ils ont posées au sol plus leurs prestations manuelles, alors que les accueillants, eux, contre-transfèrent leurs seules prestations manuelles [39]. Jusqu'à la cérémonie focale, dans la période croissante de l'ensemble cérémoniel, des dynamiques de circulation en résultent qui font que d'étape en étape, les prestations des arrivants s'additionnent pour atteindre leur plus grande quantité et complexité dans la cérémonie focale. Ceci pour chacun des deux côtés de l'ensemble cérémoniel. La dynamique incrémentale des prestations est une dimension clé du modèle qui s'articule sur la succession des étapes cérémonielles et est donc spécifique des cérémonies complexes [40]. Elle caractérise les deux côtés des ensembles cérémoniels dans les périodes croissante et décroissante. Dans leur période croissante, de totalisation - jusqu'à la fin de la cérémonie focale -, il s'agit d'une dynamique incrémentale d'augmentation, qui concerne les participants, leurs prestations, la complexité cérémonielle, l'espace et le temps du réseau. Corrélativement, dès que les prestations issues de ce processus repartent pour être redistribuées par les retours dans le réseau constitué lors de cette période croissante, les étapes de redistribution de ces prestations de retour font apparaître dans la période décroissante - de détotalisation des prestations - , une transformation qui est une sorte de renversement, par lequel le principe incrémental s'applique désormais à une diminution des prestations.

Dans une perspective large, les étapes préparatoires, la cérémonie focale, puis les étapes de redistribution, scandent l'espace et le temps sociaux de l'ensemble cérémoniel. Elles se composent en un réseau (fig. 2) couvrant les parties du pays, les groupes et les personnes concernées par les relations à élaborer.

Il faut souligner que les processus décrits ci-dessus sont indissociables d'actes et d'expériences personnelles et collectives des participants. Globalement, dans un ensemble cérémoniel, les circulations des personnes et des prestations délimitent le périmètre et I'extension de l'élaboration des relations, donc leur pratique et connaissance en extension de celles-ci. De façon plus circonscrite, quand les accueillants d'une étape de niveau 1 se rendent à une étape de niveau 2 (etc.), ils deviennent les arrivants. Chez les participants, ces permutations de positions dans les dyades font que l'expérience des rapports et relations d'appartenance ou d'extériorité et d'altérité est inversée, différenciée et modulée par la procédure cérémonielle - éventuellement plusieurs fois, ce qui inscrit chez chacun le caractère relatif de ces positionnements. Dans les cérémonies complexes, des expériences différenciées, modulées, en particulier de

\section{[38] MONNERIE 2012a.}

[39] Comme dans la forme simple, à chaque étape, les accueillants préparent, offrent et partagent un repas, ceci constitue l'importante séquence de validation des relations.

[40] C'est à dire que ce principe ne ressort pas de l'étude des formes simples, à la différence du principe dyadique, ou de celui de la validation par la préparation et le partage de repas cérémoniel (etc.), qui eux sont communs aux formes simples et complexes. 
déploiement des relations d'appartenance, ou d'extériorité et d'altérité, d'engagements, de responsabilités cérémonielles, sont ainsi systématiquement assignées à - et vécues par - un nombre considérable des participants (photos 3 et $\mathbf{4}$ ).

\section{CÉRÉMONIES DU CYCLE DE VIE ET CÉRÉMONIES DES CONFIGURATIONS SOCIALES LOCALISÉES}

Les cérémonies complexes sont ainsi constituées d'ensembles cérémoniels comportant toujours deux sous-ensembles. Un point important est que ceux-ci diffèrent de façon significative dans leurs déterminations relationnelles selon qu'on a affaire à des cérémonies du cycle de vie ou à des cérémonies des configurations sociales localisées.

Pour les cérémonies du cycle de vie des personnes adoptions, mariages, funérailles, etc. - les côtés des ensembles cérémoniels sont centrés sur les relations des personnes focales à des groupes de parenté [41] définis comme soit « paternels», soit « maternels ». J'entends par personne(s) focale(s) celles qui sont au centre de l'élaboration des relations : I'époux (et l'épouse) dans les mariages, le défunt dans les funérailles, etc. Les relations d'affinité sont ainsi l'arrière-fond structurel des distinctions dyadiques de ces cérémonies. Dans la dyade cérémonielle focale, les arrivants sont les parents maternels de la personne focale, et la relation d'altérité qui caractérise toutes les dyades cérémonielles prend donc ici une forme intime, celle d'une relation d'affinité.

Le côté maternel de l'ensemble cérémoniel est désigné dans la langue d'Arama comme « ceux qui prennent » (ajalu) car au plan quantitatif, dans la cérémonie focale, ils reçoivent en prestations transférées par le côté paternel trois fois la quantité d'objets cérémoniels qu'ils leur transfèrent. Cette asymétrie des flux de circulations caractérise les cérémonies du cycle de vie [42]. Elle est associée à la relation nommée nhoor relative aux apports respectifs de sang et de vie attribués à la mère et aux parents maternels [43]. De façon générale, plus les personnes

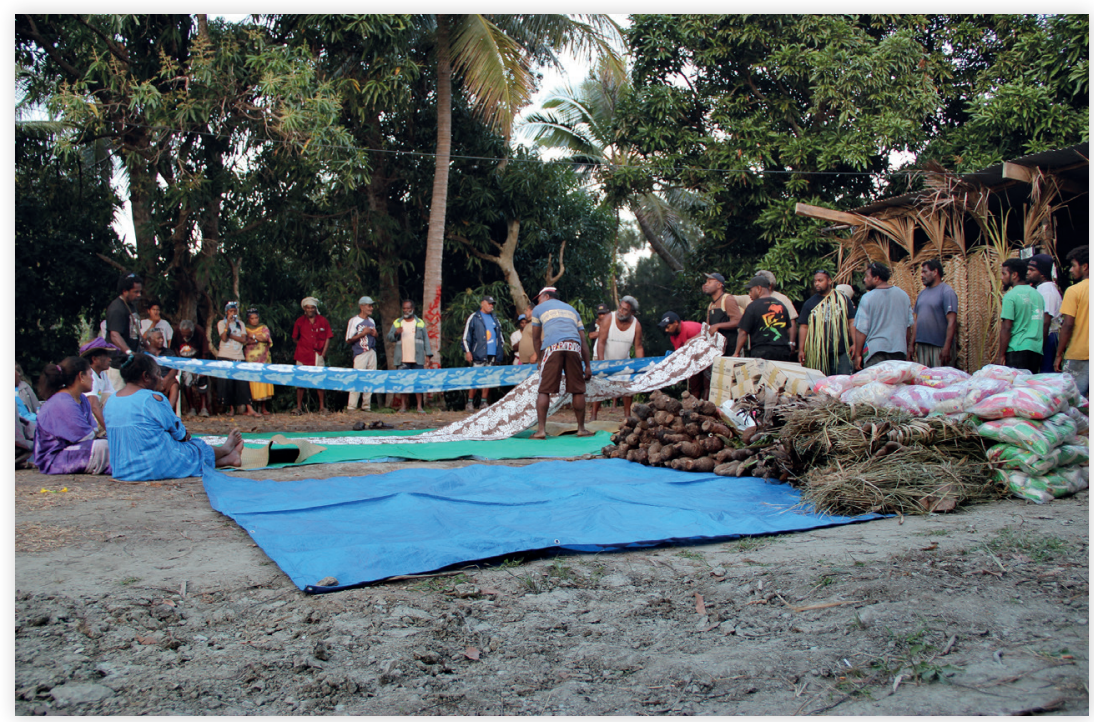

[41] Qui sont des yameevu, phwâmeevu et hulaya aju ; MONNERIE 2012b.

[42] À ma connaissance cette asymétrie vaut pour l'ensemble du monde kanak, y compris aux Iles Loyauté.

[43] Si on interprète cette relation nhoor, classiquement, comme une dette - de sang, de vie - il apparaît que la notion de dette ne configure qu'un aspect restreint des circulations cérémonielles ; les implications théoriques considérables de ce point seront traitées dans un autre contexte. Pour un phénomène comparable au plan des théories des monnaies, voir SERVET 2013.

Photo 3 : prestations dans une cérémonie focale de mariage, Tiari 2012. À droite, les représentants du côté cérémoniel de l'époux sont en train de disposer leurs prestations au sol, réparties en deux lignes (nourritures et autres objets cérémoniels) destinées au côté cérémoniel de l'épouse. À gauche, celle-ci, avec des proches, est assise au bout des lignes de prestations, face au côté de l'époux. (C) Denis Monnerie.

Photo 4 : disposition de prestations pour des funérailles, Yenghebane 1992. Des jeunes gens commencent à disposer des prestations au sol. Pendant ce temps des proches du défunt discutent avec l'orateur, un homme âgé qui porte un tee shirt vert représentant Ataï, héros de la résistance kanak à la colonisation française. (C) Denis Monnerie.

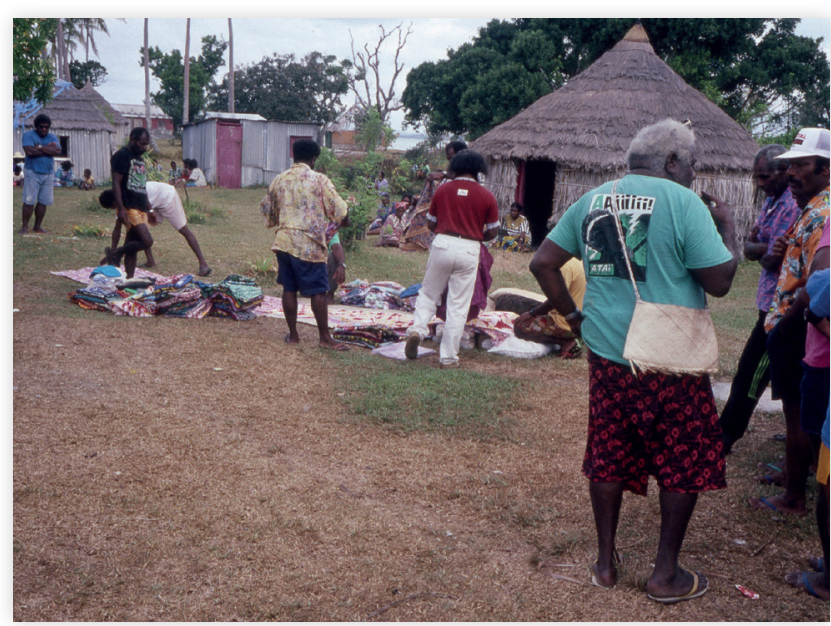




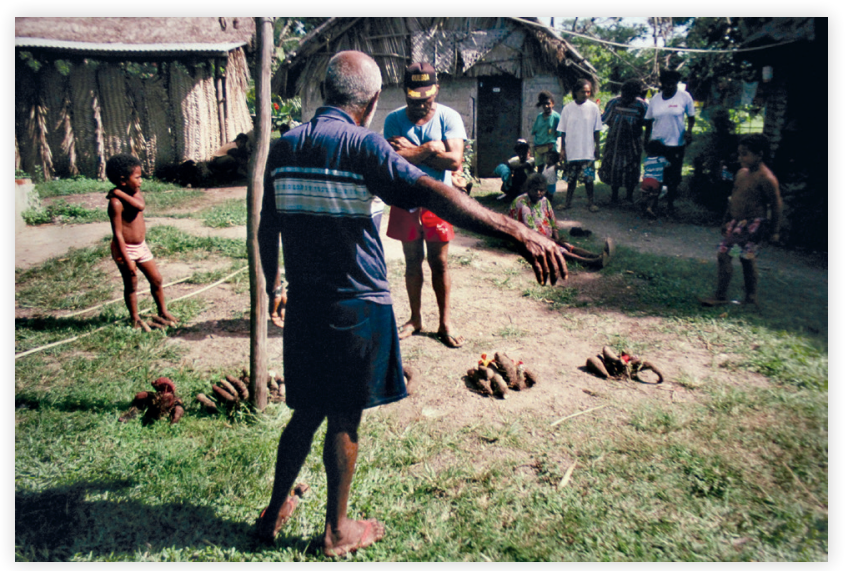

Photo 5 : cérémonie de prémices des ignames, Arama 1993. L'orateur des accueillants (de dos) fait un discours sur les tas d'ignames (associés à des fleurs d'hibiscus).

(c) Denis Monnerie.

focales concernées par ces mariages ou funérailles sont de statut important et, pour les funérailles, plus leur trajectoire d'existence est complète, plus les ensembles cérémoniels sont importants [44].

Les cérémonies des configurations sociales localisées, elles, impliquent principalement des hameaux et des sociétés locales [45] (photos 5 et 6). Par rapport au lieu de la cérémonie, le rapport entre altérité et appartenance concerne ici des relations spatiales mettant en jeu des extériorités et des altérités potentiellement très différentes. Ceci va jusqu'à impliquer des inconnus complets, comme le montre l'accueil cérémoniel fait à James Cook dans cette même région, à Balade en 1774 [46].

\section{LES OBJETS CÉRÉMONIELS}

On l'a vu, dans les procédures d'élaboration des relations, les objets cérémoniels ont une dimension indispensable de visibilité et de préhensibilité qui est mise en avant par un mythe, un aspect fondamental

[44] Principe de complexité et de complétude très répandu en Océanie et au-delà. Pour les Iles Salomon, voir MONNERIE 1996.

[45] MONNERIE 2005.

[46] COOK 1980.

[47] Pour simplifier ce texte, j'utiliserai le plus souvent le mot objet pour désigner des artefacts circulant dans les cérémonies, certains étant des plantes produites par I'horticulture, d'autres des viandes.

[48] Je souligne l'aspect institué de ces biens communs pour les distinguer d'autres comme l'eau et l'air par exemple.

[49] Par ce mot je réfère à la période 1992-2017, celle de mes recherches ethnographiques sur le terrain.

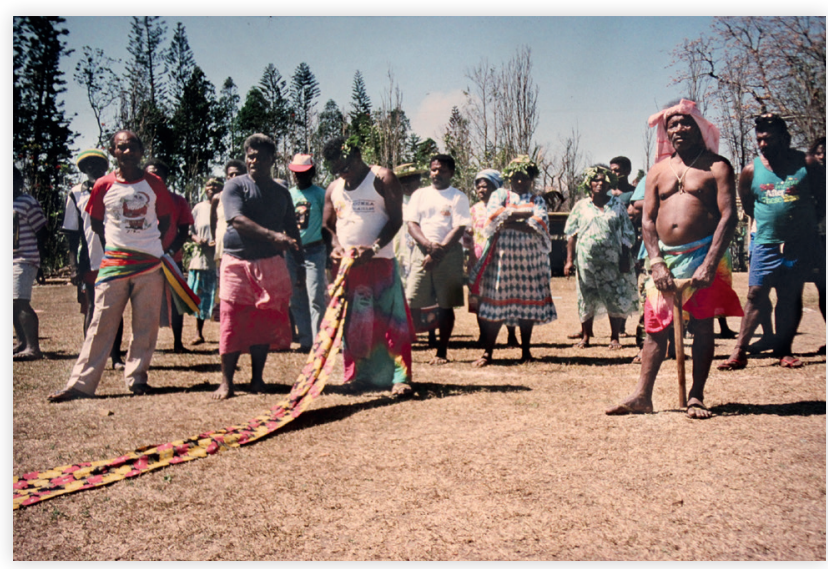

Photo 6 : grande cérémonie régionale d'arrivée et d'accueil, Bondé 1993. Le côté des accueillants : au centre, un dignitaire tient à la main une prestation d'étoffe qui lie les deux côtés cérémoniels, pendant que, sur la gauche, un autre dignitaire prononce un discours. (C) Denis Monnerie.

des conceptions locales formant la toile de fond des propositions qui suivent. Pour mieux comprendre leur rôle dans les cérémonies je commencerai par présenter les objets cérémoniels contemporains, les objets anciens auxquels ils ont été substitués et certains des enseignements de ces substitutions [47].

Dans les magasins généralistes du nord de la Kanaky Nouvelle-Calédonie, on peut acheter nombre des objets cérémoniels. Les plus caractéristiques sont les manous, pièces d'étoffes multicolores, souvent disponibles en grandes quantités. Ce n'est qu'à partir du moment où ces marchandises entrent dans les circulations cérémonielles que je viens de décrire, qu'elles deviennent des objets cérémoniels - et ce jusqu'à la fin des circulations d'un ensemble cérémoniel. Ils pourront alors être mis de côté pour être réemployés pour d'autres cérémonies, ou bien sortir du circuit cérémoniel. Il existe cependant des objets cérémoniels spécifiques. Il faut relever que, dans ces circulations, ces objets sont (re)connus de tous comme objets cérémoniels, ils sont des biens communs institués [48].

Aujourd'hui [49] dans les cérémonies du nord de Hoot ma Whaap, circulent des billets - plus rarement des monnaies kanak -, des sculptures, des artefacts spécifiquement kanak (sagaïes, frondes), des vêtements (robes mission, pantalons et chemises), des manous, des nattes, du tabac, des allumettes, des ignames (uvi) et des sacs de riz, des taros d'eau (kowe) et des sacs de sucre en poudre, des bananes et plantains, de la canne à sucre, toutes sortes de nourritures achetées en magasin (pain, farine, thé, café...), toutes sortes de viandes chassées ou élevées (cerfs, porcs, bétail, poulets) mais aussi, pour les grandes cérémonies, des gibiers cérémoniels qui pour des sociétés de bord de mer sont aquatiques (tortues 
[wan] et vaches marines [ou dugong, môdap]) et pour les autres, aériens et terrestres.

Autrefois circulaient des « haches ostensoirs », différentes monnaies kanak, des sculptures, des artefacts kanak (armes, etc.), des pagnes masculins ( $p h w i)$ et des jupes féminines (pûû) de fibres, des écorces battues, des nattes, des ignames et des taros d'eau, des bananes et plantains, de la canne à sucre, et dans les grandes cérémonies, les viandes cérémonielles déjà mentionnées. Nombre des objets contemporains sont des substituts de ces objets anciens dont bien souvent le nom est utilisé, en particulier quand les discours cérémoniels les évoquent [50]. Cependant, on observe que certains de ces objets restent utilisés. Parmi ceux-ci, les ignames, qui sont un des thèmes forts d'autodéfinition des Kanak - on souligne qu'il faut toujours en apporter au moins une dans les cérémonies. J'ai en effet rarement observé de cérémonies un peu importantes sans circulation d'ignames. Cependant, elles aussi ont un substitut : les sacs de riz. Les taros, eux, peuvent être remplacés par des sacs de sucre en poudre. À Arama, société côtière, les tortues et les vaches marines sont un cas intéressant, car elles sont au cœur de tensions entre les exigences contemporaines de protection d'espèces menacées et celles de prestige et de valorisation masculines attachées à leur capture, leur apport, leur cuisson et leur consommation [51]. Outre ces perdurations, on verra que certains objets cérémoniels contemporains sont des sortes de simulacres des objets anciens, et/ou en sont proches par leurs thèmes de signification.

\section{MODES SOCIAUX DE SIGNIFICATION DES OBJETS CÉRÉMONIELS}

Les objets cérémoniels ont des significations non verbales, relevant d'études sémiotiques, susceptibles d'être verbalisées dans les discours et les gloses.

[50] MONNERIE 2005, p. 255.

[51] MONNERIE 2016c.

[52] Dans cette région, autrefois il existait huit sortes de monnaies différentes, toutes désignées par des noms de feuilles. Aujourd'hui, on tend à traiter toutes les monnaies kanak comme identiques et à les ranger dans la catégorie hâjet. Mais dans les discours les prestations de monnaies (kanak et/ou billets) restent le plus souvent désignées par la dyade oratoire deelâ me hâjet. Le premier terme, deelâ, désigne d'anciennes monnaies qui sont glosées comme ayant un rapport à la « richesse de la parole» et servant à faire des «demandes ».
On a vu comment les opérations qui constituent les séquences de circulations distinguent, tout en les synchronisant, les transferts non verbaux des prestations d'objets cérémoniels des prestations verbales des énonciations - et écoutes - des discours. Les séquences de circulations mettent en œuvre un idiome incluant les significations non verbales des objets, des gestes de transferts et des circulations et celles, verbales, des discours, pointant le fait que les relations en cours d'élaboration combinent elles-mêmes des dimensions verbales et non verbales. Les facultés et engagements pluri-perceptuels et intellectuels des participants sont ainsi sollicités ensemble dans l'expérience cérémonielle. Il faut dissocier ces divers plans pour analyser les modes de signification des objets cérémoniels. Je prendrai trois exemples : les monnaies, les ignames et taros et les vêtements.

L'usage du terme monnaie kanak sera conservé ici. Cependant « objet valorisé » - en anglais valuable en serait une caractérisation plus pertinente, ayant pour effet de ne sous-entendre ni une dimension d'équivalent universel, ni une différence majeure, une incommensurabilité, avec les autres objets des circulations cérémonielles. Au nord de Hoot ma Whaap, les monnaies kanak les plus utilisées sont nommées hâjet [52]. Elles sont constituées d'une enveloppe végétale et/ou de tissu épais de forme rectangulaire qui est roulée, maintenue par un cordon, contenant une longueur d'une très fine cordelette sur laquelle sont enfilés de minces perles et d'autres éléments, dont de minuscules coquillages et sculptures (photo 7). Dans les séquences d'élaboration, quand elle est présentée, manipulée et transférée, la monnaie kanak est

Photo 7 : deux monnaies kanak de fabrication contemporaine (région de Hienghène), une ouverte, l'autre fermée. (c) Denis Monnerie.

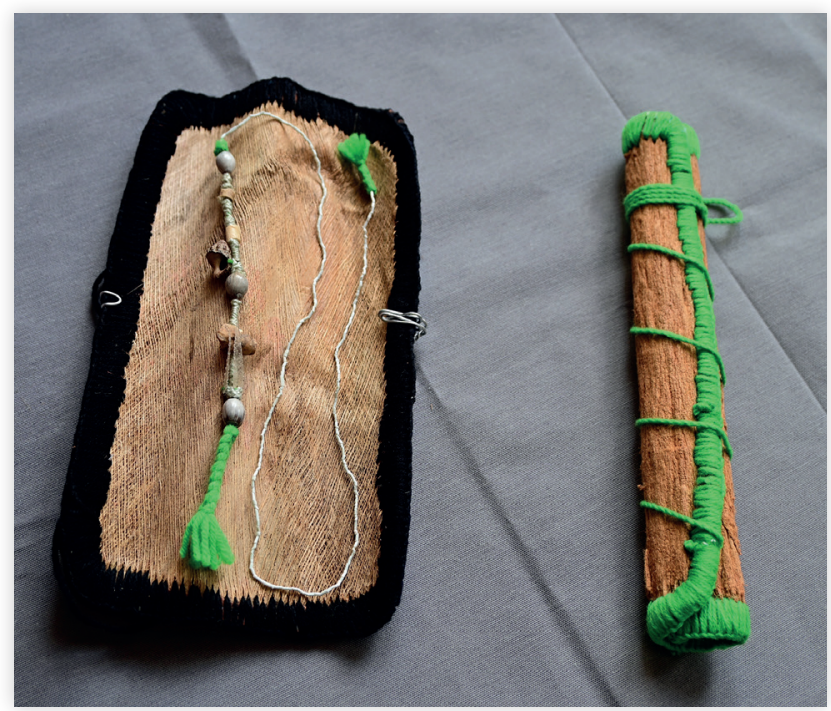


close. Ce n'est qu'au moment où elle est ouverte par le récipiendaire que son contenu est révélé. Au plan iconique ses fabrication, manipulation et observation donnent ainsi à percevoir une forme enveloppante permettant de cacher, ou montrer, un contenu. Dans les gloses et discours, cette forme est associée à des thèmes de significations et de relations associées à la reproduction humaine, autour du ventre maternel, de la grossesse et de la naissance. Son nom, hajet est glosé en résonance avec ces thèmes, comme un composé de hâ, « intérieur», et de jet, « ventre ». L'objet monnaie lui-même, et ses manipulations - présentation fermée, ouverture -, donnent lieu à des gloses qui développent ces thèmes de l'intérieur et du ventre. La gestation et la naissance sont comparées de façon récurrente à la façon dont l'enveloppe de la monnaie cache son contenu quand elle est transférée pour ne le révéler qu'au moment où on l'ouvre - et vice versa. Dans ces contextes, I'objet monnaie est conçu comme iconique d'un ventre maternel au moment d'une gestation, puis d'une naissance, qui tour à tour cachent puis révèlent le sexe du bébé, donc, pour une fratrie, un frère ou une sœur. Ceci est la figuration tactile et visuelle d'un principe général qui impliquera, à la génération suivante, l'orientation de la relation nhoor caractérisant les flux de circulations des cérémonies du cycle de vie selon qu'on s'y trouve dans la position de maternel ou de paternel (cf. supra, note 43).

Bien souvent on ne dispose pas de monnaie kanak pour une cérémonie. Le substitut en est un billet de banque plié en longueur, enveloppé dans un manou. Ce composite peut être encore complexifié par l'ajout d'un rameau feuillu, évocation des anciens noms des monnaies kanak (cf. supra). Ainsi constituée, cette prestation reproduit un certain nombre des dimensions iconiques signifiantes de la monnaie kanak, tout particulièrement l'enveloppement et la surprise potentielle concernant son contenu. On remarque que dans les deux cas la signification iconique est attachée aussi bien à une forme - enveloppante - qu'à un processus, celui de la révélation lors de l'ouverture de l'enveloppe.

On pourrait difficilement surévaluer l'importance des ignames (uvi) dans le monde kanak, qui fut même qualifié par A. G. Haudricourt de civilisation de l'igname [1964] [53]. Objet de toutes les attentions des horticulteurs et horticultrices, les ignames sont au cœur du travail conjoint des humains, de la terre et du monde. À Arama, ce tubercule ne doit pas être commercialisé, il demeure ainsi dans des circulations exclusivement kanak. Dans les cérémonies, crues, elles sont manipulées de façons spécifiques, cuites elles participent de la satiété procurée par les repas. La seule cérémonie régulière - annuelle - de cette société est la cérémonie des prémices des ignames, dans laquelle une prestation de tubercules cultivés dans chacun des hameaux les représentent comme parties constituantes de la société locale.

Les ignames constituent avec les taros d'eau une prestation cérémonielle omniprésente et les discours sont rythmés par la dyade oratoire uvi ma kowe, « ignames et taros ». Celle-ci contraste et associe des plantes aux goûts et qualités nutritives différentes avec leurs environnements différents, comme le sont leurs pratiques de culture. Elle résonne avec les distinctions-associations entre ignames masculins de culture sèche et taros d'eau féminins de culture humide. D'autres objets renvoient à ces distinctions qui sont aussi des complémentarités.

Les robes mission et les pantalons et chemises d'aujourd'hui renvoient aux jupes féminines et pagnes masculins, anciens objets de fibres dont les noms sont associés dans une autre dyade oratoire, phwi me pûû. Celle-ci pointe d'une autre façon - par des enveloppements - vers les distinctions et associations de sexe et de genre.

Dans les monnaies, tubercules et vêtements sont impliqués des rapports des objets aux relations sociales et cosmiques. Chacun articule et exprime de façon spécifique des rapports aux personnes, groupes, environnements, terroirs, substances, sexes, genres, au travail, au monde, à la vie. Avec ces objets, on perçoit l'importance du caractère pluridimensionnel des mises en relations et significations élaborées dans les cérémonies. Les objets cérémoniels sont des résonateurs périssologiques [54], mais chaque sorte est dédiée à un thème de signification spécifique. Ces thèmes peuvent se recouper. Ainsi, monnaies, tubercules et vêtements portent de façons différentes, verbales et non verbales, des significations en rapport aux distinctions-associations de sexe, de genre et aux conceptions de la vie. Les objets cérémoniels sont dotés d'agentivité spécifique : ainsi pour leurs relations à la vie, elle est directe dans le cas des ignames et taros, mais prend des formes sémiotiques diverses pour les monnaies et les vêtements. Les monnaies, par exemple, thématisent plus spécifiquement ces dimensions sur une temporalité sociale fondamentale qui s'étend du

[53] Il s'agit en fait d'une caractérisation de la configuration postérieure à la colonisation. Car auparavant, la civilisation kanak combinait, dans ses cultures et dans ses cérémonies, ignames et taros d'eau, trait qui perdure dans les prestations associant ces deux sortes de tubercules et dans la dyade oratoire uvi ma kowe.

[54] LEMONNIER 2012. 
moment crucial d'une naissance sexuée jusque dans ses projections dans la suite des générations. Ces distinctions-associations se réfractent dans les cérémonies du cycle de vie dans le face-à-face entre côtés paternel et maternel. Les associations des objets en prestations composites donnent des ampleurs supplémentaires à ces thèmes de signification.

\section{LES PRESTATIONS DANS LEURS FORMES COMPOSITES}

Les dyades cérémonielles d'objets, que redoublent les dyades oratoires, font ressortir des significations articulées en distinction-association, présentant des déclinaisons spécifiques du principe cérémoniel fondamental et englobant de la dyade.

Outre la forme dyadique, les prestations cérémonielles sont toujours des composites qui associent les différents objets mentionnés plus haut. Ces objets eux-mêmes circulent en séries qui, en fonction du principe d'augmentation incrémentale, atteignent des quantités considérables [55] lors des cérémonies focales. Ces dimensions des prestations ont un rôle central dans les procédures cérémonielles que ce soit dans les processus d'intensification ou en ce qu'elles permettent des totalisations et des fractionnements - en particulier dans les opérations d'assemblage et de comptage.

[55] Pour les plus importantes des étapes cérémonielles, on doit les transporter dans la plate-forme d'un ou plusieurs pick-ups.
Enfin, pour rassembler des idées forces de la civilisation kanak, il faut rappeler une autre dimension des circulations de ces objets : lors des séquences de transferts et contre-transferts, elles opèrent I'élaboration des relations à la manière de tressages et de nouages (photo 8).

Ces éléments généraux de mon modèle concernant les significations et circulations des objets et prestations cérémoniels sont soumis à des modulations selon le type de cérémonie et lors de chaque performance cérémonielle particulière. J'ai mentionné les quantités différentes d'objets circulant dans les côtés maternels ou paternels des cérémonies du cycle de vie. De surcroît, chaque grande sorte de cérémonie (mariage, funérailles, régionale, de prémices des ignames, etc.) implique aussi, au plan qualitatif, des pondérations différentes des objets circulants, selon le rapport de leurs thèmes de signification avec les relations élaborées par chaque performance cérémonielle particulière. Ainsi, ce modèle se module et se complexifie avec les variables que sont le type de relations impliquées, leurs formes réticulaires, leurs extensions et emprises spatio-temporelles.

\section{LES DYNAMIQUES DES FORMES CÉRÉMONIELLES COMPLEXES}

Dans la constitution progressive de chaque côté de la cérémonie focale, les étapes préparatoires de niveaux 1,2 et 3 rassemblent de façon incrémentale de plus en plus de personnes et d'objets cérémoniels qui seront transférés en grande quantité vers les étapes de niveau 4 et lors de la cérémonie focale.

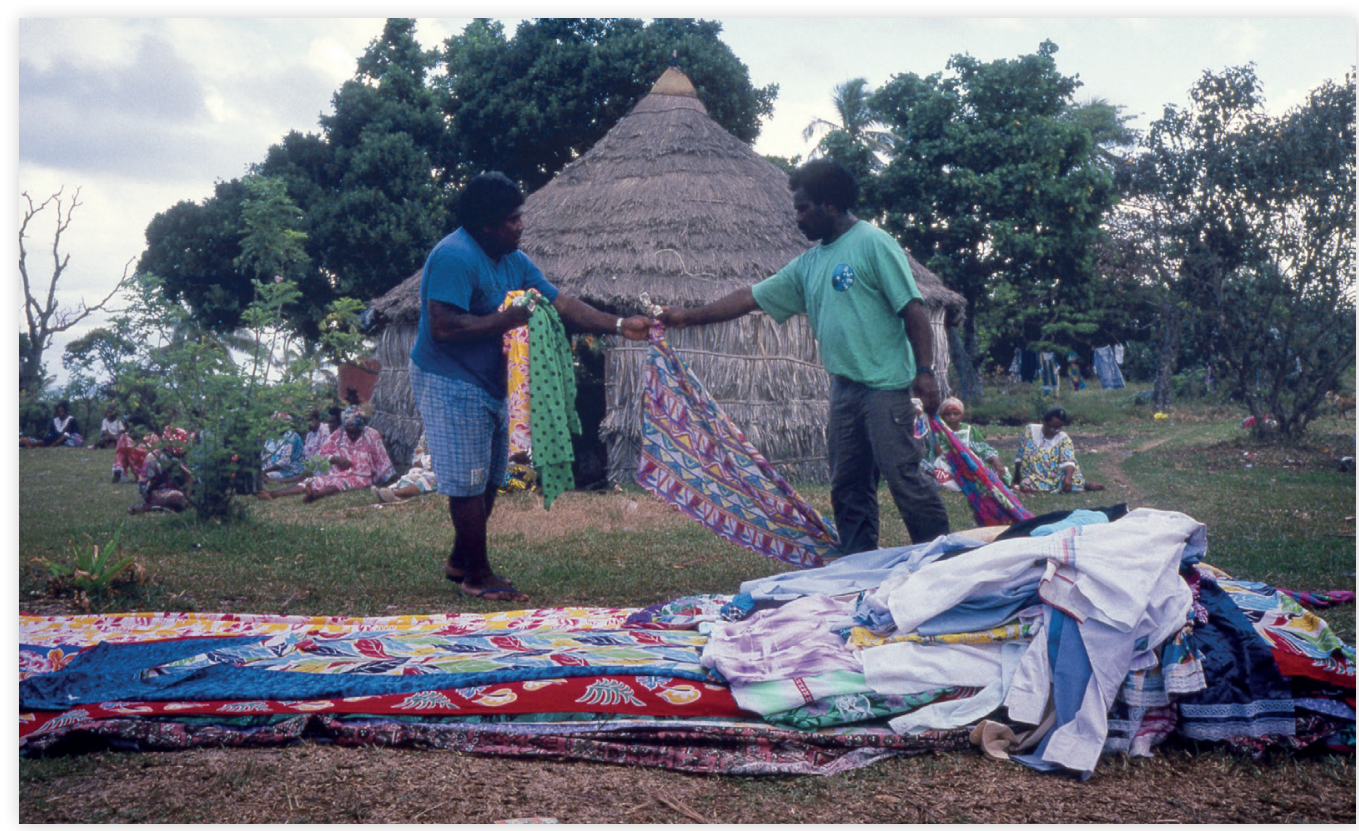

Photo 8

Transfert de prestations pour des funérailles, Yenghebane 1992 La disposition des empilements en ligne des prestations, comme la forme des prestations manuelles (cf. photo 6), soulignent graphiquement leurs rôles de liens entre les deux côtés cérémoniels. (C) Denis Monnerie. 
Le processus d'intensification incrémentale qui caractérise les procédures de constitution des deux côtés de I'ensemble cérémoniel pendant la période croissante, jusqu'à la cérémonie focale, se fonde sur les caractères composites, sériels et répétés des prestations et des opérations cérémonielles. Il se caractérise à chaque phase de chaque étape cérémonielle par la répétition et l'enchaînement de séquences de circulations de plus en plus nombreuses mobilisant de plus en plus d'objets cérémoniels dans des prestations de plus en plus considérables et des discours de plus en plus longs et de plus en plus sophistiqués.

La cérémonie focale est le rassemblement, en un lieu et temps définis à l'avance, des résultantes de ces nombreuses étapes préparatoires. Le processus opératoire d'élaboration des relations y est le plus spectaculairement développé et intensifié. Toutes les relations et tous les espaces-temps ayant contribué aux deux côtés de la cérémonie sont rendus visibles, tangibles, manipulables et transférables par les objets cérémoniels. Simultanément les relations en cours d'élaboration sont décrites et magnifiées verbalement dans de longs et parfois magnifiques discours. Alors même que tous les participants à ce large processus ne sont pas présents le jour de la cérémonie focale, tous sont à quelque degré représentés, présentifiés, avec leurs relations, par les objets qu'ils ont transférés et qui circulent et tous recevront une prestation de retour. Après cette période, croissante, de totalisation et d'intensification où les prestations seront rassemblées et réorganisées, elles vont être redistribuées pour des retours à tous ceux qui, de chaque côté, ont contribué aux cérémonies préparatoires. Des phases et séquences de redistribution interviennent comme conclusion de la cérémonie focale (photo $\mathbf{9}$ ). Les prestations de redistribution sont ensuite transférées dans un ordre précis aux ensembles contributeurs des deux côtés cérémoniels. À leur tour, leurs représentants iront au plus vite, dans les jours qui suivent, les redistribuer aux participants éloignés.

\section{LES REPAS CÉRÉMONIELS}

Toutes les cérémonies sont accompagnées de repas qui sont une importante séquence cérémonielle, principalement féminine, qui parachève et valide les relations élaborées à chaque phase, étape ou cérémonie focale. Les rapports d'acquisition des ingrédients et du bois de cuisson, de préparation des plats, de partage de commensalité et d'ingestion de nourritures cuites jouent ici un rôle majeur où s'articulent expériences personnelles et collectives de tous les participants. Avec, pour les festins des cérémonies focales, une qualité, une variété et une surabondance de nourritures très attendues, appréciées, reconnues et commentées par l'ensemble des participants - faisant la fierté des femmes qui, nombreuses, ont travaillé collectivement aux grandes cuisines (photo 10). Celles-ci sont des scènes cérémonielles collectives impressionnantes où des découpages, assaisonnements et mitonnages savants et soigneux, puis les cuissons se déroulent parallèlement à la scène où des hommes opèrent des transferts et contre-transferts verbaux et non verbaux et qui se tient à quelque distance et que j'ai plus particulièrement décrite et modélisée dans ce texte. Le travail culinaire rejoint le travail de circulation [56] et

[56] Une part considérable de la scène cérémonielle des cuisines est elle aussi sous-tendue par des relations d'affinité.

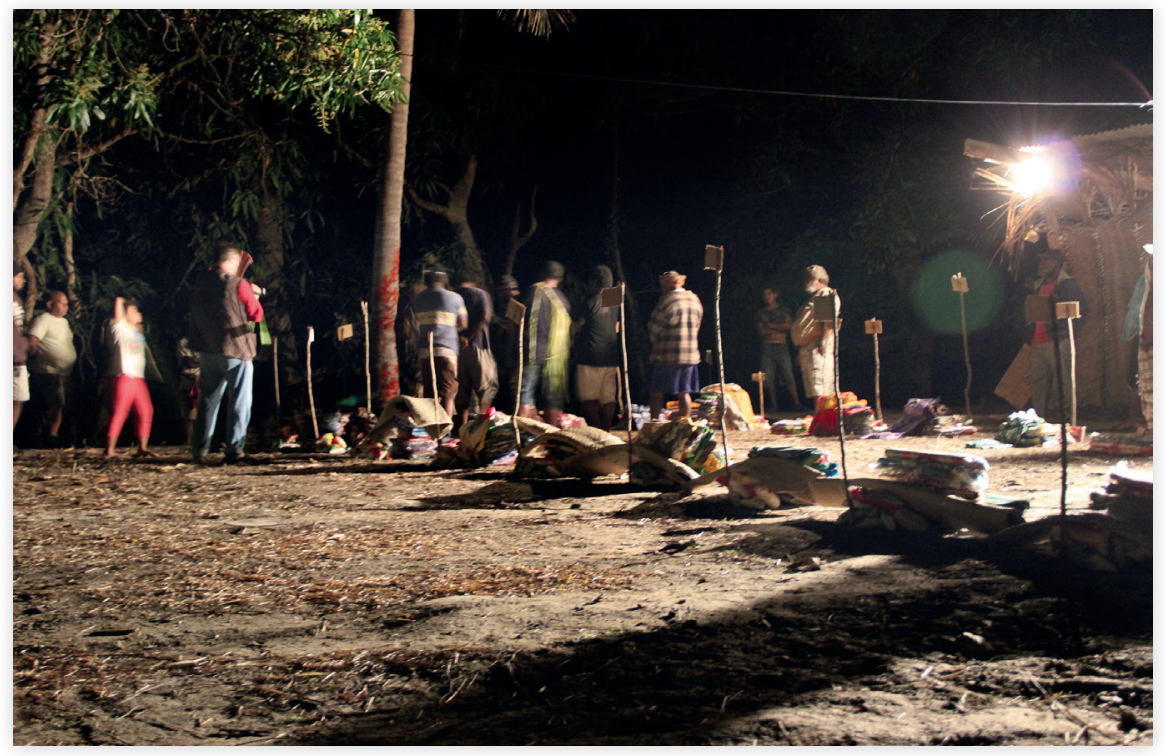

Photo 9

Moment de redistribution à Tiari en fin de cérémonie focale de mariage, 2012. Chaque tas d'objets cérémoniels est une prestation de retour destinée à un collectif ayant contribué à la cérémonie focale. Leurs noms, qui sont aussi énoncés dans l'ultime discours de cette cérémonie, sont écrits sur des étiquettes placées au sommet de perches plantées dans le sol. Ces prestations doivent ensuite être redistribuées au plus vite vers l'ensemble des groupes et personnes ayant contribué aux prestations du collectif nommé ici. (c) Denis Monnerie. 


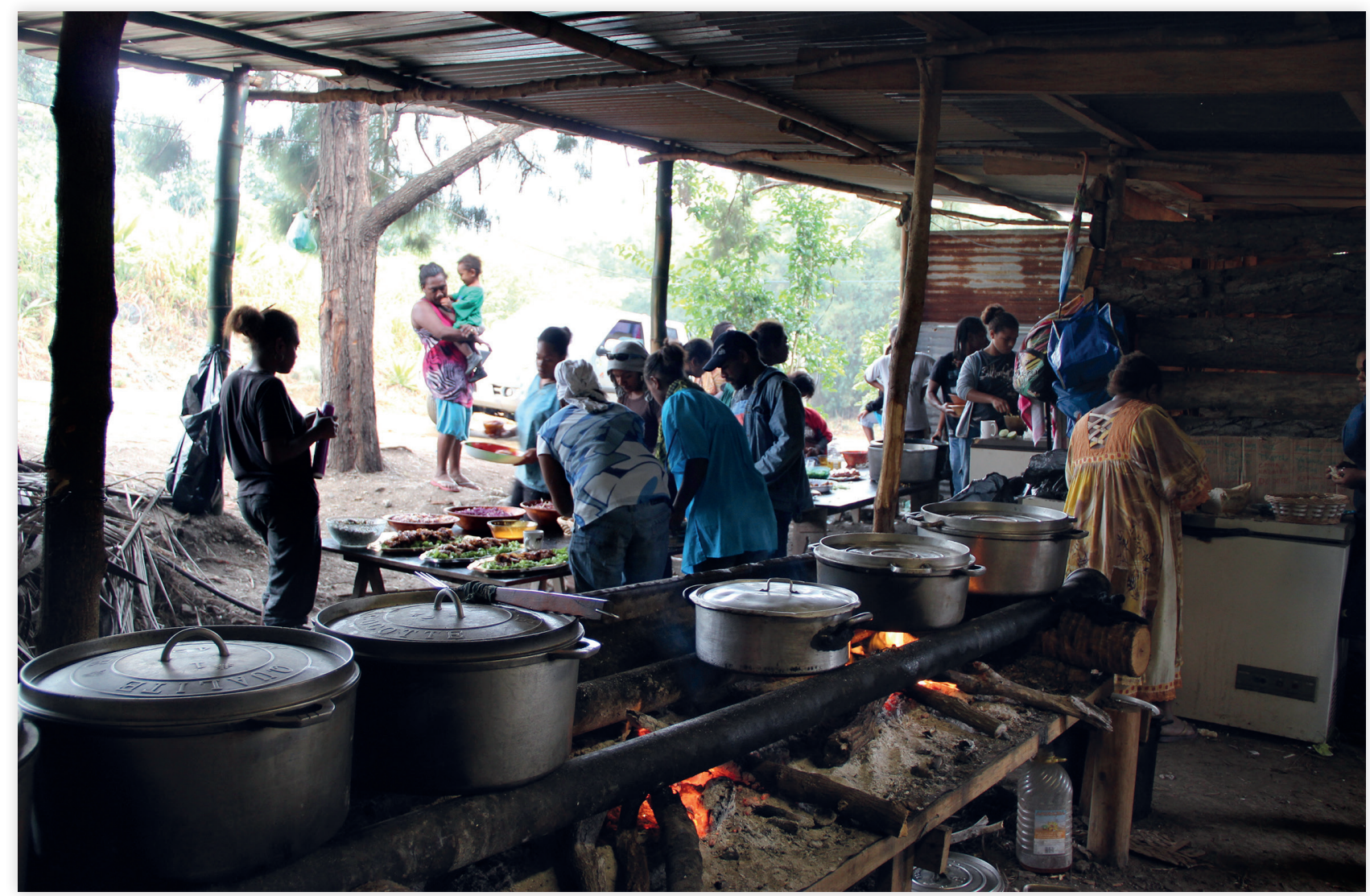

Photo 10 : cuisine dans une cérémonie focale de mariage, Tiari 2012. Au premier plan les grosses marmites en fonte d'aluminium disposées sur deux rails, chacune avec des mets différents, cuisent au feu de bois. Au fond, des femmes et quelques hommes mettent la dernière main aux entrées. (c) Denis Monnerie.

ils se complètent et se valident dans la préparation, la commensalité, le partage, l'ingestion de nourriture (photo 11). Ces repas visent à la satiété des participants, dimension de fortification immédiatement perceptible de l'intensification de la vie, à la fois personnelle et collective qui résulte de l'ensemble du travail cérémoniel.

\section{CONCLUSION}

Les cérémonies kanak sont accomplies en des espacetemps largement distribués faisant vivre et exprimant les emprises réciproques des relations sociales sur le pays et du pays sur ces relations. Ces formes intensifiées du social que sont les cérémonies participent ainsi à des institutions centrales de la socialité et de la souveraineté du monde kanak. Elles se caractérisent par une mise en place dyadique où des distinctions des deux côtés, de l'ordre de l'appartenance, ou de I'altérité et de l'extériorité, sont graduées et sont articulées par des procédures prescrites. Celles-ci opèrent I'élaboration de relations par des circulations de personnes, d'objets et de discours suivies de leur validation dans un repas partagé. Présents dans la forme cérémonielle minimale d'accueil, ces principes de base portent en eux des développements à formes quasi fractales pouvant générer des ensembles cérémoniels considérables. J'ai proposé le concept d'ensemble cérémoniel pour souligner le fait crucial que la plupart des cérémonies un peu importantes ne se limitent pas à leur seule cérémonie focale et que, pour en constituer les deux côtés, des étapes préparatoires sont réparties dans deux sous-ensembles.

J'ai montré comment les objets cérémoniels kanak sont des éléments cruciaux de ces procédures cérémonielles en tant que biens valorisés reconnus de tous et institués. Ils sont manipulés dans des opérations prescrites de transfert et contre-transfert qui décrivent graphiquement des nouages et tressages de relations, synchronisés avec des discours scandés. Leur mise en circulation dans des prestations composites se poursuit étape cérémonielle après étape cérémonielle, jusqu'à la cérémonie focale, puis dans les prestations de retour. Jusqu'à la cérémonie focale, leur circulation suit un principe d'augmentation incrémentale d'étape en étape, qui s'accompagne de la complexification de tous les actes cérémoniels. Un principe quasi symétrique, mais inversé, de diminution, préside aux circulations des prestations de retour. 
Les objets cérémoniels ont des qualités à la fois relationnelles, substantielles et significatives, mettant en œuvre des agentivités spécifiques. Leurs qualités relationnelles impliquent l'inclusion dans des prestations composites de séries d'objets du même type avec des réorganisations répétées de leurs agencements dans les prestations, dans des circulations et dans leurs moments de nouages et de tressages. Leurs qualités substantielles sont liées à leur « matérialité », qui est plutôt conçue par la civilisation kanak en termes de visibilité, de préhensibilité et, pour les nourritures, de qualités reproductives, gustatives et roboratives. Le tout est associé aux significations non verbales complexes portées par ces objets qui se combinent avec celles, verbales, des gloses fréquentes faites à leur sujet, de leurs noms et des discours cérémoniels. Chaque sorte d'objet cérémoniel porte ainsi des thèmes de significations larges, qui entrent en jeu dans un idiome cérémoniel où se développent et s'entrecroisent la rhétorique verbale des discours et celle, non verbale, des compositions de prestations différenciées selon les sortes de cérémonies et leurs réalisations particulières. Si on observe les objets cérémoniels à travers les actes de leurs manipulations, ils manifestent leur agentivité d'abord en ce qu'ils sont porteurs de l'engagement de chaque participant de l'ensemble cérémoniel. Ces objets sont ensuite en quelque sorte anonymisés dans les circulations de l'ensemble cérémoniel, jusqu'au moment où chaque participant reçoit une prestation de retour attestant simultanément de son engagement initial et de l'accomplissement collectif de la cérémonie et de l'élaboration des relations. C'est ainsi que, traversant l'ensemble cérémoniel, les objets ont porté les actes relationnels de chaque participant tout en agissant en tant que biens communs institués dans l'élaboration collective des relations.

Photo 11

Repas dans une cérémonie focale de mariage, Tiari 2012. Aujourd'hui chaque mariage tend à se distinguer par des couleurs spécifiques - ici du bleu - qui se retrouvent dans la décoration des salles de repas et souvent dans les

vêtements des adjoints à qui sont dévolues les tâches culinaires. Après les grandes phases de la cérémonie, sur des bancs et de longues tables, le plus souvent associés en fonction de leurs relations et de leur âge, les convives partagent des festins très attendus où se succèdent des dizaines de plats différents. La socialité des cérémonies focales est toujours très intense ; les mariages se distinguent en ce qu'elle y est aussi très décontractée, très exubérante. (c) Denis Monnerie.

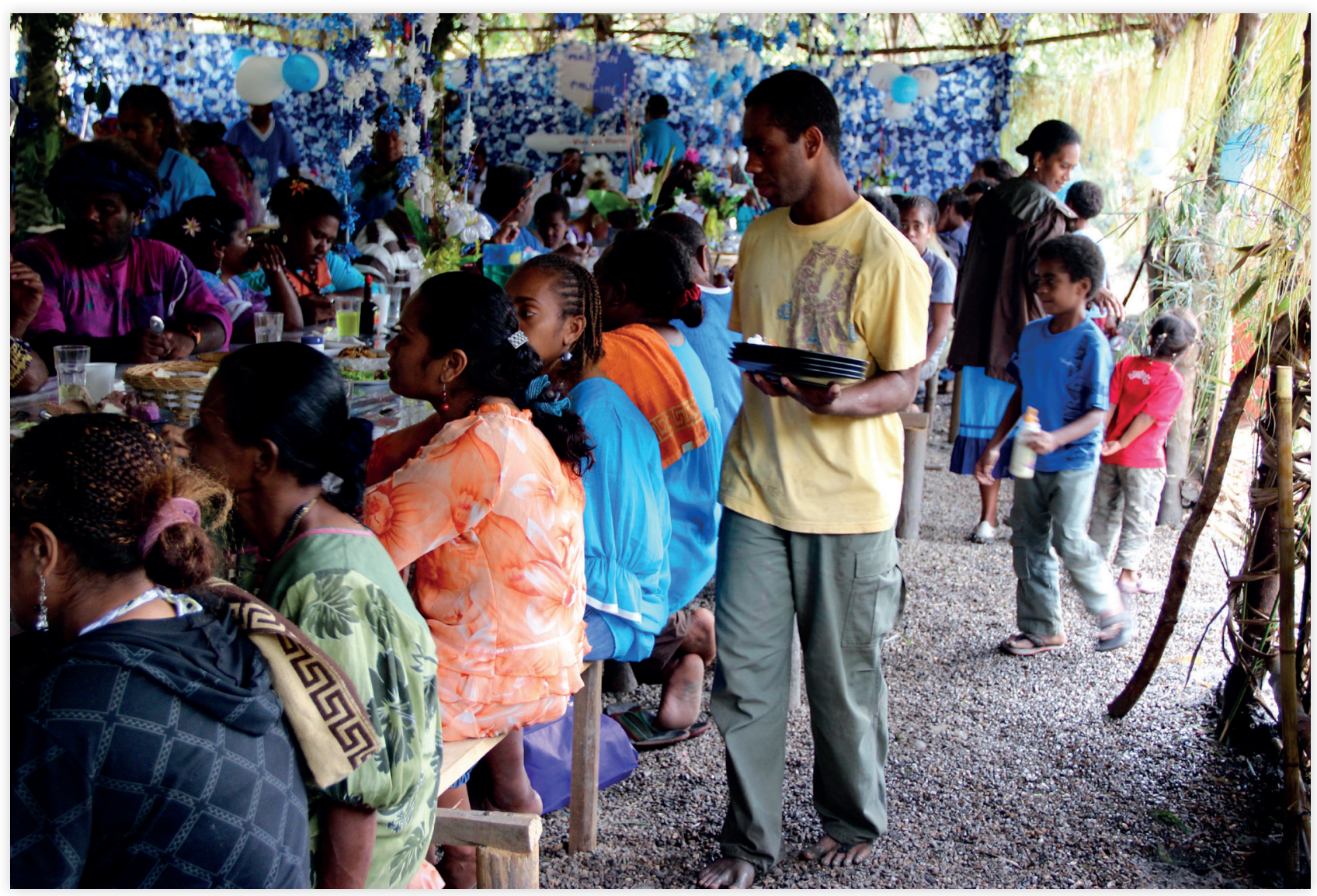


Bretteville, Dominik, 2019, «L'os et le souffle » ou les ancêtres porteurs de vie. Le système social et cosmique des Paimboa (Nouvelle-Calédonie), Paris.

Chave-Dartoen, Sophie \& Monnerie, Denis, à paraître, Receiving and Intergrating. The Other Side of Insular Mobilities. A Comparative Approach for Melanesia and Polynesia, Pacific Islands (UISPP series).

CooK, James, 1780, Relations de voyages autour du monde. Paris (1980).

CoPPET, Daniel de, 1981, « The life-giving death » dans S. Humphreys \& H. King (éd.), Mortality and Immortality, London. CoupaYe, Ludovic, 2013, Growing Artefacts, Displaying Relationships. Yams, Art and Technology amongst the Abelam of Papua New Guinea, New York - Oxford.

Dumont, Louis, 1978, « La Communauté anthropologique et l'idéologie », L'Homme 18/3-4, p. 83-110.

GAGNIÈRE, M., 1905, Étude ethnologique sur la religion des néo-calédoniens, Saint-Louis.

GeLL, Alfred, 2009, L'Art et ses agents. Une théorie anthropologique, Paris.

Godin, Patrice, 2015, Les échanges sont le souffle de la coutume. Logiques sociales de la «vie» et de la «puissance» en pays hyeehen (Nouvelle-Calédonie, côte nord-est), Thèse, Université de la Nouvelle-Calédonie, Nouméa.

GrAFF, Stéphanie, 2015, Autodétermination et autochtonie en Nouvelle-Calédonie. L'effacement progressif de la question coloniale, Thèse de doctorat, Institut de Hautes Études Internationales et du Développement, Genève.

HerTz, Robert, 1908, «Contribution à une étude sur la représentation collective de la mort », Sociologie religieuse et folklore, Paris (1970).

ILlouz, Charles, 2010, La parole ou la vie. Valeur et dette en Mélanésie, Rennes.

LAMBert, Pierre, 1900, Moeurs et superstitions des néo-calédoniens, Nouméa.

Leblic, Isabelle, 2004, « Métissage et parenté. Assimilation de non-Kanak dans le système des moitiés matrimoniales à Ponérihouen », Annales d'histoire calédonienne 1. «La Nouvelle-Calédonie. Terre de métisssage », p. 35-56.

Leblic, Isabelle, 2008, Vivre de la mer, vivre avec la terre... en pays kanak. Savoir et techniques des pêcheurs kanak du sud de la Nouvelle-Calédonie, Paris.

Leblic, Isabelle, 2010, Échanger dans la route. Parenté, alliances, rituels et territoires paicî à Ponérihouen (NouvelleCalédonie), Manuscrit pour I'HDR, EPHE, Paris.

LeEnhARDT, Maurice, 1937, Gens de la Grande Terre, Paris.

Lemonnier, Pierre, 2005, « L'objet du rituel : rite, technique et mythe en Nouvelle-Guinée », Hermès 43/3, p. 121-130.

Lemonnier, Pierre, 2012, Mundane objects. Materiality and Non-Verbal Communication, Walnut Creek.

LeRoI-GourhaN, André, 1965, Le geste et la parole. II La mémoire et les rythmes, Paris.

MALINOWSKI, Bronislav, 1922, Argonauts of the Western Pacific, London.

MAUsS, Marcel, 1923-1924, «Essai sur le don », dans Sociologie et anthropologie, Paris (1950), p. $142-279$.

Monnerie, Denis, 1996, Nitu, les vivants, les morts et le cosmos selon la société de Mono-Alu (Iles Salomon), Leiden.

MonnerIe, Denis, 2005, La parole de notre Maison. Discours et cérémonies kanak aujourd'hui. Paris.

Monnerie, Denis, 2008, «The Great House and the Marché. Two Kanak exchange complexes (New-Caledonia) », dans P. Stewart \& A. Strathern (éd.), Exchange and Sacrifice. Durham, N.C., p. 27-54.

MonnerIe, Denis, 2012a, « Objets cérémoniels, chaînes opératoires et élaboration des relations sociales (Arama et Hoot ma Whaap, Kanaky Nouvelle-Calédonie, Mélanésie) », dans M. Bailly, P. Lemonnier \& S. Revolon (éd.), Objets irremplaçables, Thema de Techniques et Cultures 58, p. 122-141. www.cairn.info/load_pdf.php?download=1\&ID ARTICLE=TC 058 MonnerIe, Denis, 2012b, «Significations, valorisations et performativité de la nomination dans l'organisation sociale à Arama (Nouvelle-Calédonie). Système, résilience et créativité », dans S. Chave-Dartoen, C. Leguy \& D. Monnerie (éd.), Nomination et organisation sociale, Paris, p. 129-165.

Monnerie, Denis, 2014a, «Expériences, performances et compétences dans les cérémonies kanak (Arama et Hoot ma Whaap, Kanaky Nouvelle-Calédonie) », dans S. Bornand \& C. Leguy (éd.), Compétences et performances, Paris, p. 265-290. Monnerie, Denis, 2014b, « Les échanges en Océanie et l'anthropologie », dans L. Dousset, B. Glowcewski \& M. Salün (éd.), Les sciences humaines et sociales dans le Pacifique Sud. Terrains, questions et méthodes, Marseille, p. 127-159.

Monnerie, Denis, 2016a, « Relations et substances : l'anthropologie et les enseignements des cérémonies kanak. (Nord de la Grande Terre, Kanaky Nouvelle-Calédonie) », dans C. Barraud, A. Iteanu \& I. Moya (éd.), Puissance et impuissance de la valeur, I'anthropologie comparative de Louis Dumont, Paris, p. 235-260.

Monnerie, Denis, 2016b, «Oceania and the regional relations paradigm: contrasting regional networks and beyond. 177-190 », dans F. Valentin \& G. Molle (éd.), Spatial dynamics in Oceania, Séances de la Société Préhistorique Française 7, Paris. http://www.prehistoire.org/515_p_48142/acces-libre-sEance-7-la-pratique-de-I-espace-en-ocEanie-decouverte-appropriation-et-emergence-des-systemes-sociaux-traditionnels.html 
Monnerie, Denis, 2016c, «Résistances à la patrimonialisation. Agentivités, créativités et souveraineté kanak (Arama, Nouvelle-Calédonie) », dans Aurélie Condevaux, Géraldine Le Roux \& Laura Schuft (éd.), Du corps à l'image. La réinvention des performances culturelles en Océanie, Journal de la Société des Océanistes 142/3, p. 53-72.

MonnerIe, Denis, 2018, « Subjuguer ou décrire ? Repenser la puissance coloniale et comprendre les résistances et résiliences kanak en Nouvelle-Calédonie », dans Émilie Nolet, Peter Lindenmann \& Laurent Dousset (éd.) Médiations politiques en Mélanésie contemporaine, Marseille, p. 125-175. https://www.pacific-credo.fr/index.php/fr/pacific-credo-publications PeIRCE, Charles S., 1978, Écrits sur le signe, Paris.

Pillon, Patrick, 1995, « Parenté agnatique et par alliance, positions statutaires et circulation des offrandes : le déroulement contemporain d'une cérémonie des morts dans la vallée de la Kouaoua (Nouvelle-Calédonie) », Journal de la Société des Océanistes 100-101, p. 165-190.

Servet, Jean-Michel, 2013, « Monnaie : quand la dette occulte le partage », Revue Française de Socio-Économie 12/2, p. $125-147$.

THURNWALD, Richard, 1937, L'économie primitive, Paris.

TURNeR, Victor, 1968, Les tambours d'affliction, Paris (1972).

Van Gennep, Arnold, 1909, Les rites de passage, Paris (1981). 